Rev. Elev. Méd. vét. Pays trop., 1966, 19, 3, (277-282).

\title{
Note sur le cycle biologique de Macracanthorhynchus hirudinaceus (Pallas 1781) à Madagascar
}

\author{
par P. DAYNES
}

\begin{abstract}
RÉSUMÉ
L'auteur décrit le cycle de $M$. hirudinaceus réalisé à Madagascar avec pour Hote intermédiaire Bricoptis voriolosa (cetonjinae) et pour Hote définitif le porc, Sus scrofa domesticus. II donne des chiffres obtenus en ce qui concerne les chronologies évolutives chez l'Hote intermédiaire et chez I'Hote définitif.
\end{abstract}

\section{INTRODUCTION}

L'Acanthocéphalose du porc, due à Macrocanthorhynchus hirudinaceus, est une infestation relativement fréquente à Madagascar. Dans certaines régions plus de 7 p. 100 des porcelets sont reconnus, par la coproscopie, porteurs d'Acanthocéphales (2), le pourcentage d'animaux réellement infestés étant nettement supérieur. Les porcins sont parfois fortement parasités et présentent sur l'intestin des lésions très prononcées aux lieux de fixation des helminthes. C'est ainsi que nous rencontrons souvent des jeunes porcs de 15 à $30 \mathrm{~kg}$, porteurs de 30 à 40 acanthocéphales. Signalons le cas d'un porcelet pesant $5 \mathrm{~kg}$ en très mauvais état et mort de cachexie. Il était porteur de 52 acanthocéphales.

L'Acanthocéphalose, bénigne en général dans les infestations paucl-parasitaires hors les rares cas de perforation intestinale, est loin d'être négligeable dans des cas semblables où elle entraîne retards de croissance et amaigrissements pouvant conduire souvent à la mort.

ANTIPIN (1) estime que l'issue fatale est le lot de 30 p. 100 des animaux infestés dans les régions où cette parasitose prend une allure enzootique.

Le traitement de cette helminthose est actuellement des plus aléatoires aussi l'étude du cycle évolutif est-elle intéressante tant du point de vue biologique que du point de vue prophylactique.

\section{Rappel de l'évolution.}

L'évolution de $M$. hirudinaceus classiquement décrite nécessite un hôte intermédiaire.

Elle est la suivante:

Le porc infesté expulse dans le milieu extérieur les embryophores pondus dans son tube digestif par la femelle fécondée. Ces embryophores sont ingérés par des «vers blancs》, formes larvaires de divers coléoptères scarabeidae en général.

Selon les régions les insectes suivants ont été reconnus comme étant les hôtes intermédiaires possibles de $M$. hirudinaceus (4) (6) (8).

\section{Europe :}

Melolontha melolontha Scarabeidae

\section{Melolontho hippocostani -}

Cetonia aurato

Liocola brevitorsis

Amérique du Nord :

Phyllophaga rugosa

Lochnosterna armata

Xyloryctes satyrus

Cotinis nitida 
Amérique du Sud :

Phoenurus splendidus

Gromphos lacordairei

Asie :

Gymnopleurus vupsus

Horpalus tridens

Blatella germanica mûre de $M$. hirudinaceus récoltée quelques heures auparavant sur un porc infesté.

Cette femelle étant découpée en tous sens à l'aide d'une paire de ciseaux le liquide qui s'écoule contient des embryophores mûrs et des embryophores immatures (vérification faite au microscope entre lame et lamelle). Une goutte de ce liquide est déposée à la pipette au niveau des pièces buccales du ver blanc tenu délicatement entre pouce et index.

Nous avons ensuite disséqué, au bout de laps de temps plus ou moins longs, des larves d'insectes provenant des élevages infestés.

Lorsque des acanthelles ont été rencontrées elles ont été récoltées et ont pu servir à des essais d'infestation de porcs neufs.

Ces porcs neufs, provenant d'un élevage indemne d'acanthocéphalose, contrôlés par coproscopie, ont été élevés en cages individuelles cimentées, à l'abri des insectes. Certains d'entre eux ont été conservés comme témoins. Les porcs expérimentalement infestés, autopsiés au bout d'un délai variable devaient être porteurs d'acanthocéphales alors que les témoins élevés dans les mêmes conditions mais non infestés devaient apparaître, à l'autopsie, exempts de parasites.

Nos prospections entomologiques nous ont mis en présence de 4 sortes différentes de vers blancs qui, pour autant que l'on puisse en juger à ce stade, représentaient quatre espèces.

Les 4 variétés de larves rencontrées ont permis de réaliser 4 lots différents supposés contenir chacun une espèce différente d'insecte. Deux lots étaient réalisés avec des larves melolonthoides typiques faisant penser que l'on avait affaire à des larves de Melolonthinae et deux lots contenaient des larves ayant l'aspect morphologique de larves de Cetoniinae.

Le diagnostic des espèces en cause est particulièrement difficile sur les larves.

Chaque lot devait contenir des larves de la même espèce pour que l'obtention (souhaitée) d'un Imago dans un lot permette alors d'étendre son identification à tout le lot. Chaque lot a donc fait l'objet d'une récolte en un seul lieu comme en un seul temps et toutes les larves $y$ étaient apparemmen identiques.

\section{Résultats expérimentaux.}

Ces quatre élevages furent conduits avec des bonheurs différents, Les deux premiers lots 
(larves de Melolonthinde) survécurent très peu de temps. Les larves moururent en quelques jours à quelques semaines au plus, Nous n'avons jamais retrouvé d'acanthelle chez ces melolonthinae larvaires. Aucun individu n'ayant afteint le stade Imago nous ne savons pas quelles étaient les espèces en cause.

Un des deux lots de cetoniinae nous permit d'obtenir le stade l'mago máis pas plus les larves que les stades Imago ne furent trouvés porteurs d'Aconthelles.

L'espèce en cause était Chromoptilia diversipes (Cetoniinoe).

L'élevage du dernier lot (larves de cetoniinae) nous permit de suivre l'évolution des acanthelles, d'infester des porcs et d'obtenir des stades Imago de l'insecte (Identifié par la suite comme Bricoptis varioliosa).

Cependant, cet élevage insuffisamment bien conduit a vu' s'installer une mortalité importante, Celle-ci atteignait en fin d'expérience 90 p. 100 des individus. Il faut noter qu'au cours de cet élevage environ 15 p. 100 des larves formèrent leur coque nymphale mais peu aboutirent au stade Imago. Nous avons pu disséquer 10 larves dont trois ne parurent pas infestées et 4 adultes dont deux étaient infestés. Nous ne citerons les dates que pour les larves reconnues infestées.

Les vers blancs en cause ont été récoltés le 1/2/65. Ils ont été infestés le 2/2/65.

Les dissections et autopsies suivantes ont été effectuées :

a) Le 25/3/65 un ver blanc disséqué présente dans sa cavité générale, au niveau du tube digestif antérieur, des kystes renfermant des ocanthelles à différents stades d'évolution. En prenant pour référence les six stades d'évolution de l'Acanthella décrits par KATES (6), le stade $V I$ étant le stade infestant, on note :

$\begin{array}{rrrr}7 & \text { acanthelles au stade } & \text { I } \\ 11 & - & - & \text { II } \\ 2 & - & - & \text { III } \\ 7 & - & - & \text { IV } \\ 10 & - & - & \text { V } \\ 5 & - & - & \text { VI }\end{array}$

Il est bien entendu qu'il n'y a pas de solution de continuité entre ces stades caractéristiques de l'évolution qui est continue.

Le classement ci-dessus est approximatif. les acanthelles étant classées dans le stade de développement dont elles se rapprochent le plus

Entre la date d'infestation du ver blanc et celle de la dissection la température moyenne de la pièce où se trouvait l'élevage était de l'ordre de $24^{\circ} \mathrm{C}$.

Les acanthelles récoltées ont été conservées mais n'ont pas servi à une infestation expérimentale de porc!

b) Le 26/3/65 une larve disséquée montre la présence de dix acanthelles se répartissant comme suit :

3 acantheiles ont un degré d'évolution les situant entre les stades I et II.

3 acanthelles se sifuent approximativement au stade IV.

1 acanthelle en est approximativement au stade $V$.

1 acanthelle se situe entre les stades $V$ et $V l$.

2 acanthelles sont apparemment au stade $V I$.

Ces 10 acanthelles servent à infester per os le pore no 1 A qui sera autopsié le 30/6/65 sans avoir présenté d'embryophore d'acanthocéphale dans ses excréments.

L'autopsie nous permet cependant de trouver deux jeunes Macracanthorhynchus fixés dans l'intestin grêle.

c) Le 6/5/65 une larve d'insecte infestée montre à la dissection la présence de 4 acanthelles en voie de différenciation et se repartissant ainsi :

3 acanthielles proches du stade II.

1 aconthelle située entre les stades IV et $V$.

Le porc n०2 A auquel sont administrées, per os, ces 4 acanthelles ne sera porteur d'aucun acanthocéphale lors de son autopsie le 1/9/65.

d) Le 7/5/65 la dissection d'un ver blanc permet la récolte de cing acanthelles dont une au stade IV et quatre au stade VI.

Le porc $n^{\circ} 3$ A est infesté avec ces 5 acanthelles. Son autopsie pratiquée le 2/9/65 mettra en évidence, fixés dans le duodenum, deux Macrocanthorhynchus, un jeune mâle et une femelle immature.

e) Le $8 / 5 / 65$ douze acanthelles sont trouvées à la dissection d'une larve d'insecte. Elles se répartissent comme suit : 
2 acanthelles au stade $V$.

9 acanthelles au stade VI.

1 acanthelle située entre ces deux stades et très légèrement mobile.

Sous la loupe il est possible de voir, en sérum physiologique, le rostre de cette dernière acanthelle s'invaginer lentement.

On fait absorber ces 12 acanthelles au porc no 4 A. Il sera autopsié le 24/9/65. Pas plus que les autres porcs i! n'aura permis de voir des embryophores d'acanthocéphale dans ses excréments. Cependant, il est porteur de 2 acanthocéphales $O^{\prime}$ et de $4 \%$ qui paraissent mûres. Nous effectuons de nouvelles coproscopies très soignées sur les excréments de ce porc. Par la méthode de concentration par sédimentation nous finissons par trouver de très rares embryophores d'acanthocéphales dans un champ microscopique. On peut en déduire qu'au moins une des femelles d'acanthocéphale est bien mûre.

f) Le 2/7/65 deux larves d'insecte sont disséquées. Elles contiennent l'une deux acanthelles, l'autre trois acanthelles, toutes les cinq d'allure très avancée et avec des crochets brun foncé. Ces acanthelles ne servent pas à infester un porc et sont conservées.

g) Le $27 / 9 / 65$ les porcs no $5 \mathrm{~A}$ et $n^{0} 6 \mathrm{~A}$ de même origine que les précédents et élevés dans les mêmes conditions afin de servir de témoins sont sacrifiés et qutopsiés. Aucun des deux n'est porteur d'Acanthocéphale.

h) Le $6 / 11 / 65$ nous obtenons dans notre élevage un premier stade Imago vivant qui naus permet enfin' une identification de l'espèce d'insecte en cause. II s'agit de Bricoptis variolosa (cetoniinae). Cet insecte, disséqué après identification, n'était pas porteur d'acanthelle.

Le même jour dans deux coques nymphales nous trouvons deux adultes morts, également identifiés par I'I, R. S. S. M. comme Bricoptis variolosa.

i) Le $15 / 12 / 65$ le même élevage nous permef d'obtenir 3 Imago de Bricoptis variolosa. Deux de ces 3 B. voriolosa sont infestés d'acanthelles très évoluées (stade $\mathrm{VI}$ ) : 9 acanthelles chez un insecte ef 4 chez l'autre.

Des porcs neufs choisis comme précédemment sont infestés.

Le porc no 1 B est infesté avec les 9 acanthelles du premier insecte et le porc no 2 B est infesté avec les 4 acanthelles du deuxième insecte.

Ces porcs sont mis en élevage dans les mêmes conditions que précédemment ainsi que 4 porcs témoins $(3 \mathrm{~B}, 4 \mathrm{~B}, 5 \mathrm{~B}$ et $6 \mathrm{~B})$.

Le $17 / 2 / 66$, le porc na 2 B est autopsié; il se montre porteur de 2 acanthocéphales, un mâle de $7 \mathrm{~cm}$ et une femelle de $18 \mathrm{~cm}$.

Le 18/2/66 le porc no 1 B est autopsié ; il est porteur de six Macracanthorhynchus, 3 males et 3 femelles. Les mâles mesurent près de $7 \mathrm{~cm}$ alors que les femelles mesurent de 19 à $23 \mathrm{~cm}$.

Les 18 et 19/2/66 les porcs témoins no3 B, $4 B$.

$5 \mathrm{~B}$ et $6 \mathrm{~B}$ sont autopsiés et se montrent dépourvus d'acanthocéphale.

En résumé sur 6 porcs infestés par nous 5 ont été trouvés porteurs d'Acanthocéphales alors que 6 porcs témoins en ont été reconnus exempts.

\section{Conclusion ef discussion.}

Le cycle de l'Acanthocéphale du porc, Macraconthorhynchus hirudinaceus, a donc été fermé à Madagascar, grâce à Bricoptıs variolosa comme hôte intermédiaire.

Chaque fois que l'on a fait ingérer par un porc des acanthelles infestantes on a vu se développer des acanthocéphales dans son tube digestif, alors que dans le même temps et dans les mêmes conditions les témoins non infestés restaient indemnes de parasites.

Un porc infesté avec des acanthelles insuffisamment évoluées n'a pas fait d'Acanthocéphalose (porc no 2 A).

On remarquera d̀ ce propos que les vers blancs infestés présentaient des Acanthelles à différents stades d'évolution. Cela est il dô à des vitesses 'différentes d'évolution des Acanthelles chez un miême hôte, à une maturité variable des embryophores infestants ou à des réinfestations successives des vers blancs? N'oublions pas nos conditions d'infestation : embryophores puisés chez une femelle mûre ; possibilité pour le ver blanc d'absorber des embryonphores d'acanthocéphales disséminés dans son milieu d'élevage ou collés sur son corps.

Notre expérience nous a permis de trouver une durée d'évolution chez l'hôte intermédiaire de moins de 2 mois : infestation positive du 
porc no $1 \mathrm{~A}$ après évolution de l'Acanthelle chez l'hôte intermédiaire du 2/2/65 au 26/3/65 soit pendant 52 jours.

Nous avons également pu noter une durée d'évolution chez l'hôte définitif correspondant à la période, prépatente de l'infestation : chez le porc no 4 A il s'est écoulé un délai de l'ordre de 4 mois $1 / 2$ pour que l'Acanthelle infestante se transforme en adulte sexuellement mûr et se reproduisant.

Nous avons constaté la persistance del'infestation par $M$. hirudinaceus du stade larvaire des insectes au stade imago. C'est à ce stade que nous avons pu identifier Bricoptis varioloso (cetoninae) comme hôte intermédiaire.
Notons que plus de la moitié des Acanthelles infestantes (stade V) administrées aux porcs se sont développées en acanthocéphales adultes puisque nous en avons retrouvés soit 2 sur 4 (porc no $3 \mathrm{~A}$ et $2 \mathrm{~B}$ ) soit 6 sur 9 (porc no $4 \mathrm{~A}$ et $1 \mathrm{~B}$ ) soit même 2 sur 2 (Porc no $1 \mathrm{~A}$ ).

Enfin cette expérience nous aura permis de concevoir des améliorations pour nos élevages de larves d'insectes ce qui nous sera utile pour poursuivre cette étude sur une plus grande écheile.

\section{Institut d'Elevage et de Médecine Vétérinaire des Pays Tropicaux, \\ Laboratoire central de l'Elevage, Tananarive}

\title{
SUMMARY \\ Note on the biological cycle of Macrocanthorhynchus hirudinaceus \\ (Pallas 1781) in Madagascar
}

\begin{abstract}
The cycle of $M$. hirudinoceus in Madagascar is described. The intermediate host has been shown to be Bricoptis variolosa (cetoniinae) and the definitive host is the pig Sus scrofa domesticus. Figures from various'chronological stages of evolution in the intermediate and definitive host are given.
\end{abstract}

\section{RESUMEN}

Noła sobre el ciclo biológico de Macracanthorhynchus hirudinaceus (Pallas 1781) en Madagascar

El autor describe el ciclo de $M$. hirudinaceus. Lo realizó experimentalmente en Madagascar con Bricoptis variolosa (cefoninae) como huesped intermediario y con el cerdo, Sus crofa domesticus, como huésped definitivo. Nota datos concernientes a los estados de evolución en el huésped intermediario y en el definitivo.

\section{BIBLIOGRAPHIE}

1. ANTIPIN (D. N.) in ERSHOV (V. S.). Parasitology and Parasitic Desease of Livestock 1956. Troduit du Russe par israel Program of Scientific Translation, 1960.

2. DAYNES (P.). - Note sur les Helminthoses des animaux domestiques reconnues à Madagascar. Rev. Elev. Méd. Vét. Pays Trop. 1964, 17, 3, 477, 490.
3. DOLLFUS (R. Ph.). - Etude Morphologique et Systématique de deux espèces d'Acanthocéphales parasites de Lémuriens et de Singes. Ann. Porasit. Hum. Compo. 1938, XVI, 385, 419.

4. EUZEBY (J.), - Les maladies vermineuses des animaux domestiques, 1963. Tome I, Fascicule 2. 
5. HURPIN (B.) in BALACHOWSKY (A. S.). Traité d'Entomologie appliquée à l'Agriculture. 1962, Tome I. Premier Volume.

6. KATES (K. C.). - Development of the Swine Thorn-Headed Worm, Macracanthorhynchus hirudinaceus, in its intermediate Host. Am. J. Vet. Rev. Res. 1943, 5, 173, 181.

7. KATES (K. C.). - Am. J. Vet. Res. 1944, 5, 166.
8. ONO (S.). 一 J. Jap. Soc. Vet. Sci. 1933, 12, 61 et suiv.

9. VAN CLEAVE (H. J.). - Developmental stages in Acanthocephalan life history. All Union Leningrad Acad. Agricult. Sci. Moscow 1937-739, 7.44.

10. VAN CLEAVE (H. J.). - A critical review of terminology for immature stage in Acanthocephalan life histories. J. parosit. 1947. $33,2,118,125$. 